\title{
Clinical relevance of an unfused pancreatic duct system
}

\author{
C. J. MITCHELL, ${ }^{1}$ D. J. LINTOTT, W. S. J. RUDDELL, M. S. LOSOWSKY, AND \\ A. T. R. AXON
}

From St. James's University Hospital, and the General Infirmary at Leeds

SUMMARY In man, the main pancreatic duct is normally derived from ventral and dorsal embryological buds of the pancreas. In a minority of people, failure of fusion of the two buds results in separate drainage of the dorsal and ventral pancreas, so that the accessory duct provides the main drainage for the gland. Patients with this anomaly demonstrated at endoscopic retrograde pancreatography (ERP) have been investigated to assess whether non-fusion of the main pancreatic duct predisposes to the development of pancreatitis. A failure of fusion of the pancreatic ducts was seen in 21 out of $449(4.7 \%)$ successful pancreatograms; four of these 21 patients had definite clinical evidence of pancreatitis and two patients had possible pancreatic disease, but in the remainder the anomaly was not considered to be clinically relevant. An abnormal pancreatogram suggesting pancreatitis was present in 116 out of the 428 patients $(27 \cdot 1 \%)$ with a normally fused duct system. The anomaly was found as frequently in the whole series as it was seen in patients with pancreatitis. These findings suggest that embryological failure of pancreatic duct fusion does not predispose to the development of pancreatitis. However, the presence of this anomaly may lead to misinterpretation of ultrasonographic and CT scan findings.

The pancreas is formed embryologically from distinct ventral and dorsal buds which arise from the duodenal diverticulum after rotation of the ventral bud and have fused by the seventh week of life (Meckel, 1812). Wirsung (1642) first demonstrated the main pancreatic duct in man and Santorini (1775) accurately described the duct system and demonstrated the accessory pancreatic duct.

Normally, the main pancreatic duct is derived from both embryological parts of the pancreas; the dorsal pancreas provides the main duct in the tail and body of the gland with the ventral anlage providing the main duct in the head of the gland. The accessory duct (of Santorini) is the remaining portion of the duct in the dorsal pancreas which may drain through an accessory papilla more proximally in the duodenal loop.

It is thus not surprising that anomalies of the pancreatic duct system occur and their frequency has been documented in large systematic necropsy studies by Baldwin (1911), Howard and Jones (1947), Rienhoff and Pickerell (1954), Kleitsch (1955), and Berman et al. (1960). The duct of Wirsung is the

${ }^{1}$ Address for correspondence: Dr C. J. Mitchell, Department of Medicine, St. James's Hospital, Leeds LS9 7TF.

Received for publication 16 May 1979 major pancreatic duct in $90 \%$ of specimens and an accessory duct of Santorini is present in $85 \%$ with communication between the two ducts in $33-90 \%$ of specimens.

In a minority of cases $(7 \%)$, the accessory duct provides the main drainage system. Infrequently, the two ducts fail to fuse, with the duct of Santorini $(1-7 \%)$ providing the main drainage for the gland. It is this anomaly which is the subject of this communication.

The development of endoscopic retrograde pancreatography (ERP) has enabled demonstration of the pancreatic duct system in large numbers of patients. When the duct system is not fused, only the ventral duct is demonstrated by cannulation of the main ampulla (Figs. 1 and 2). Previous reports by Cotton and Kizu (1976) and Gregg (1977) have shown that pancreatitis was present in a high proportion of patients with an unfused pancreatic duct system and suggested that the anomaly might be a cause of pancreatitis. However, Rösch et al. (1976), Kruse (1977), and Thompson et al. (1978) found that pancreatitis was present in only a minority of their patients with this anomaly. None of these studies has compared the incidence of pancreatitis in patients who have an unfused duct system with the incidence seen in patients with normal pancreatic anatomy demonstrated at ERP. 
We report a series of patients in whom a failure of fusion of the two ducts was demonstrated at ERP who have been studied retrospectively. In order to assess whether the anomaly found could be relevant to the pathogenesis of pancreatitis, we have also compared these patients with those with pancreatitis and normal pancreatic duct anatomy demonstrated at ERP.

\section{Methods}

An Olympus JF-B duodenoscope was used to perform ERP with injection of $65 \%$ Urografin (Schering Chemicals Ltd.) under television control. The pancreatograms were assessed to identify patients with an unfused pancreatic duct system and to determine the number of pancreatograms in which pancreatitis was demonstrated in a normally fused duct system. The criteria used for diagnosing pancreatitis from the pancreatograms were those described by Ashton et al. (1978).

Since the criteria for detecting pancreatic disease in patients with non-fusion of the pancreatic ducts were the clinical, biochemical, and operative findings, the patients with a normal duct system and pancreatitis at ERP were also assessed using the same criteria.

Lundh tests were performed using a standard method (Mottaleb et al., 1973). Mean tryptic activity

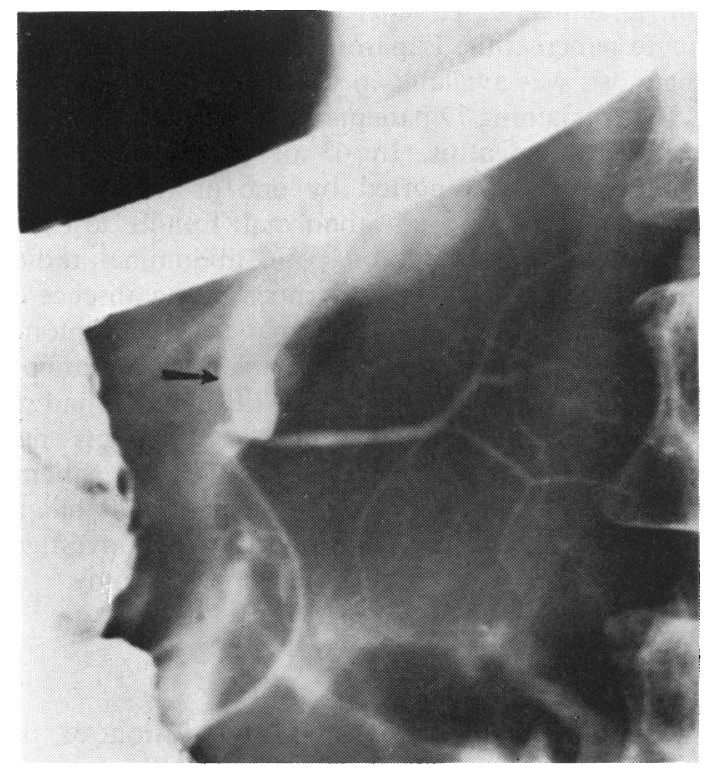

Fig. 1 Pancreatogram of an unfused ventral pancreatic duct system. The common bile duct (arrowed) which also drains through the main ampulla, has been opacified as well.

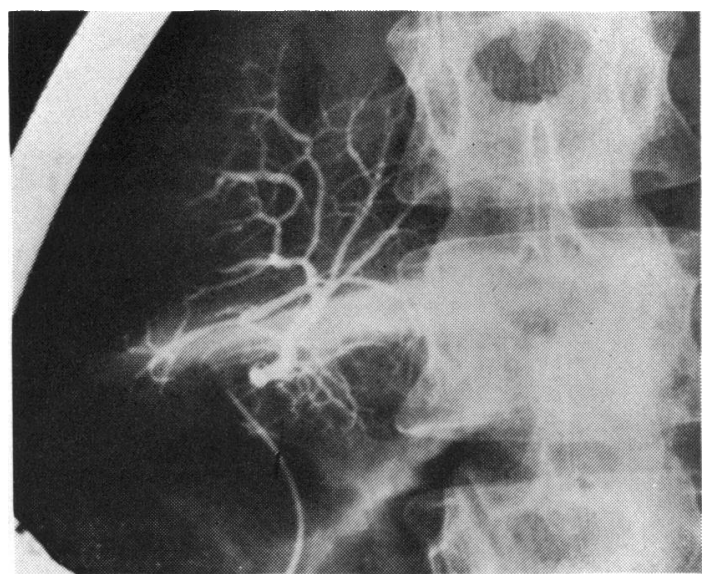

Fig. 2 The pancreatogram of a more extensive unfused ventral pancreatic duct system.

(MTA) was measured by the method of Wiggins (1966) and the lower limit of normal in our laboratory is $9 \cdot 7 \mu$ equiv $/ \mathrm{ml} / \mathrm{min}$.

\section{Results}

PATIENTS WITH UNFUSED

PANCREATIC DUCTS

Out of 449 consecutive patients in whom ERP was successful, there were 21 patients with a separate ventral pancreatic duct system. Details of patients are summarised in the accompanying Table. The unfused duct system showed abnormalities suggestive of pancreatitis in only one patient, and was normal in the remaining 20 patients.

Four patients had definite clinical evidence of pancreatitis and we were unable to find a cause for this, such as bilary tract disease or alcohol abuse. The first patient (L.G.) gave a two year history of epigastric pain which was not relieved by cholecystectomy; full gastrointestinal investigation was normal, except for an impaired Lundh test MTA without pancreatic steatorrhoea, and she continues to have recurrent attacks of epigastric pain. The second patient (J.S.) has had three severe episodes of acute pancreatitis with serum amylase levels up to 6000 Somogyi $\mu / \mathrm{ml}$ and laparotomy confirmation of the diagnosis on one occasion; a Lundh test was abnormal shortly after the last attack, but she has remained well on follow-up for another 12 months. The third patient (A.B.) presented with diarrhoea and weight loss and was found to have an abnormal Lundh test and arteriographic signs suggestive of pancreatitis; serum amylase levels, glucose tolerance, and faecal fat excretion were normal. The fourth patient (D.M.) had a three year history of recurrent abdominal pain associated with marked hyper- 


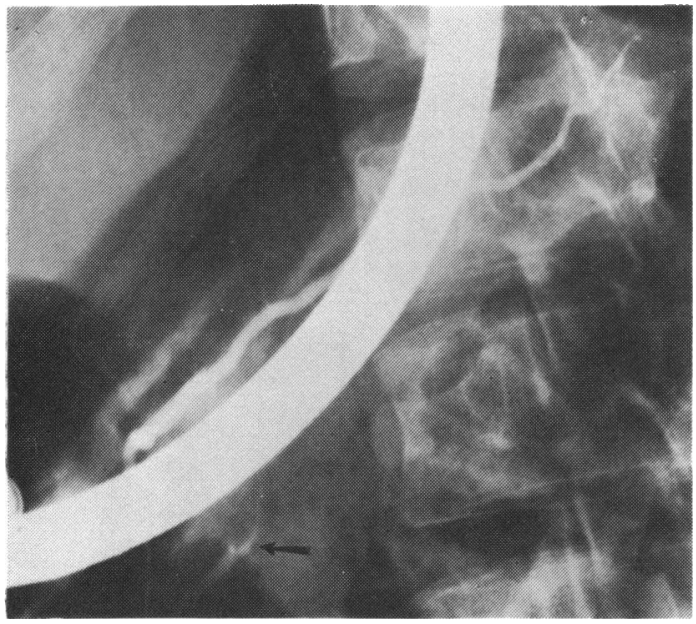

Fig. 3 In this patient the main pancreatic drainage through the accessory ampulla has been demonstrated. Some residual contrast remains in the unfused ventral pancreatic duct (arrowed) from prior cannulation of the main ampulla.

amylasaemia on four occasions; this patient was found to have signs of pancreatitis in the unfused ventral duct system at ERP but has no evidence of pancreatic insufficiency.

In another two patients, the evidence for the presence of pancreatic disease is inconclusive. In one of the patients (F.R.) the finding of an abnormal Lundh test may not be significant, as the patient was severely ill at the time and had previously undergone extensive small bowel resections for Crohn's disease; this was the only patient in whom we were able to demonstrate a normal duct system from the accessory ampulla (Fig. 3) and, although he made some response to therapy with pancreatic supplements, his gross steatorrhoea was more dramatically improved by a low fat diet with MCT supplements. The other patient (F.W.) was referred for ERP from another hospital; he had drunk considerable quantities of alcohol in the past and developed diabetes in the preceding year. After a cerebrovascular accident, he had an episode of severe upper abdominal pain, which was considered on clinical grounds to be due to pancreatitis, but serum amylase levels could not be measured during the acute attack and he has since had a normal Lundh test.

In the remaining 15 patients the finding of an abnormal pancreatic duct system did not seem relevant. Thirteen patients have become asymptomatic after either cholecystectomy (six), treatment with a high fibre diet (four), withdrawl of drug therapy (two) or spontaneously (one); one patient has been lost to follow-up (although she was well when last seen) and another patient continues to have epigastric pain in spite of full gastrointestinal investigations.

Serum amylase levels were normal in all these 15 patients, six of whom had a normal pancreas at laparotomy and a Lundh test was normal in six patients. In five patients neither laparotomy nor a Lundh test had been performed; all of these five patients became asymptomatic on follow-up and an adequate explanation for their illness was found in four patients.

\section{PATIENTS WITH NORMAL PANCREATIC} DUCT ANATOMY

ERP was successful in 449 patients. Out of 428 patients with normal pancreatic duct anatomy, a normal pancreatogram was seen on 270 occasions and evidence of pancreatic carcinoma was present in an additional $\mathbf{4 2}$ patients.

Signs of pancreatitis at ERP were seen in 116 out of the 428 patients $(27 \cdot 1 \%)$ with a completely fused duct system. The aetiology of pancreatitis was bilary tract disease (47 patients), alcohol abuse (14 patients), idiopathic (51 patients), drug-induced (two patients), traumatic (one patient), or familial pancreatitis (one patient).

Acute pancreatitis was considered to be present in 39 of the 116 patients on the basis of episodes of severe upper abdominal pain with marked hyperamylasaemia, 25 patients having suffered recurrent acute pancreatitis. Laparotomy confirmation of the diagnosis was available in six patients.

The remaining 77 patients were considered to have chronic pancreatitis. In 63 of these patients the diagnosis was supported by one or more of the following criteria: an abnormal Lundh test (39 patients); calcification on plain abdominal radiography (11 patients); steatorrhoea in the absence of small bowel or liver disease (16 patients); laparotomy findings ( 22 patients). In the remaining 14 patients the diagnosis of chronic pancreatitis at ERP had no other confirmation; four of these patients had grossly abnormal pancreatograms (two patients were alcoholics) and the other 10 patients with biliary tract disease were referred to us either for investigation of jaundice or endoscopic sphincterotomy.

\section{Discussion}

In this study we have found non-fusion of the pancreatic duct system in 21 out of 449 successful pancreatograms $(4.7 \%)$. This compares with an incidence of $1.3-6 \%$ (overall $3.7 \%$ ) for this anomaly in previous reports of pancreatic duct abnormalities 
found at ERCP (Phillip et al., 1974; Rösch et al., 1976; Gregg, 1977; Kruse, 1977; Thompson et al., 1978). Cannulation of the accessory ampulla is technically extremely difficult and it was possible to achieve this on only one occasion.

It is difficult to assess the significance of nonfusion of the pancreatic ducts in relation to the development of pancreatic disease because ERCP is performed upon a highly selected group of patients. Necropsy studies indicate an incidence of $1-7 \%$ for this anomaly (Baldwin, 1911; Howard and Jones, 1947; Rienhoff and Pickerell, 1954; Kleitsch, 1955; Berman et al., 1960), so that the frequency found in this study is that which might be expected in a normal population.

In this study, the incidence of pancreatitis in the group with non-fusion of the pancreatic ducts $(19 \%)$ was similar to that found in patients with a normally fused duct system $(27 \cdot 1 \%)$, although analysing the data in this way may merely reflect the selection of patients for ERCP. An alternative method of assessing the results is that out of 449 patients in whom the pancreatic duct system was demonstrated, there were $21(4 \cdot 7 \%)$ patients with an unfused duct system, whereas out of 120 patients with pancreatitis by clinical and/or ERP criteria, there were four patients $(3.3 \%)$ with an unfused duct system. Thus, pancreatitis with abnormal duct anatomy was seen no more commonly than the duct anomaly was found at ERP. Likewise, no significant differences between the groups emerged if the 14 patients were excluded in whom evidence of pancreatitis was found only at ERP. On the basis of our data it is therefore not possible to implicate nonfusion of the pancreatic ducts as a cause of pancreatitis.

In contrast with this study, several authors have suggested that the anomaly might be a cause of pancreatitis. Cotton and Kizu (1976) and Gregg (1977) found that the incidence of pancreatitis in these patients was $58 \%$ and $45 \%$ respectively. Heiss and Shea (1978) reported four patients with unfused pancreatic duct systems and pancreatitis but give no details of the incidence of the anomaly found at ERP. Kruse (1977) found that $20 \%$ of his patients with unfused pancreatic ducts had pan-

Table Details of patients

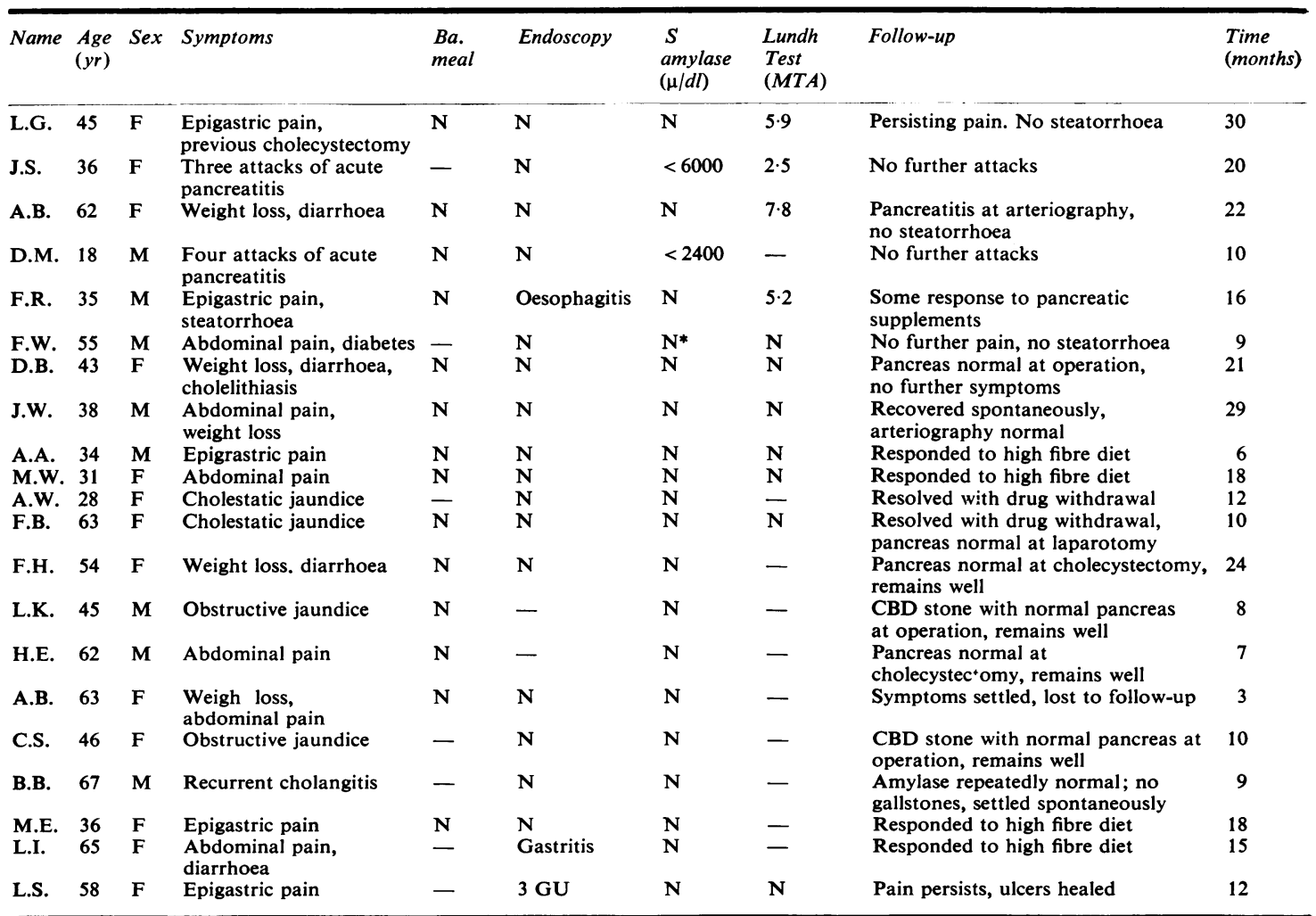

* Not measured in acute attack. 
creatitis and suggested that the anomaly might be causative, because this was a higher incidence than that found in the normal population; however, we have encountered a higher incidence of pancreatitis at ERP in patients with a normally fused duct system.

Other authors have also found that only a minority of patients with unfused pancreatic systems had pancreatitis; Rösch et al. (1976) quoted an incidence of $19 \%$ and Thompson et al. (1978) found that only one of their 11 patients had definite evidence of pancreatitis. In none of the reports quoted above is any information given as to the incidence of pancreatitis at ERP in patients with normally fused duct systems (which is considerable in our experience), so that it is difficult to interpret their findings.

Another problem in assessing the patients with an unfused ventral pancreatic duct is that we have been able to demonstrate the main drainage of the pancreas (via the accessory ampulla) in only one patient. These patients could therefore still have abnormalities of the main pancreatic duct which have not been detected, although in previous reports the presence of pancreatitis in these patients has been detectable by standard methods other than ERP. We have tried to overcome this problem by assessing these patients using clinical follow-up, amylase levels, Lundh test results and operative findingsthe only methods available if other morphological tests are unreliable in this situation (see below). Using these criteria, we feel that we have excluded or detected the presence of pancreatic disease in most of the 21 patients with non-fusion of the pancreatic ducts. In the remaining five patients who had not previously had a Lundh test or laparotomy, we did not feel that additional investigation was justified in this retrospective study, as they had all become asymptomatic and a reasonable explanation for their symptoms had been found. For comparison, the same criteria have been applied to patients with pancreatitis and normal duct anatomy at ERP as to the patients with non-fused duct systems.

The relevance of pancreatic duct anomalies to the pathogenesis of pancreatitis must remain debatable at present. Berman et al. (1960) suggested that the presence of a communicating accessory duct might reduce the incidence of pancreatitis by acting as a safety valve when the main duct was obstructed. This suggestion is supported to some extent by Mairose et al. (1978) who found a three-fold increase in the incidence of hyperamylasaemia after ERCP in patients with an absent accessory pancreatic duct.

An additional point of particular interest is that four of the patients with an unfused ventral pancreatic duct system were referred for ERP because enlargement of the head of the pancreas had been diagnosed by grey-scale ultrasonography (three) or CT scanning (one). In none of these patients was any other evidence found to suggest the presence of pancreatic disease and it is presumed that the findings at ultrasonography and CT scanning were due to the non-fusion of the pancreatic ducts that was found to be present. It may therefore be important to identify the duct anomaly so that falsely abnormal results shown by morphological scanning techniques can be identified. Two of the patients with unfused ducts had pancreatic arteriograms and these showed no vascular anomalies.

This study suggests that non-fusion of the pancreatic duct system is not a factor in the pathogenesis of pancreatitis and does not support previous suggestions to the contrary. However, a conclusive answer could be provided only by performance of ERP in a consecutive series of patients presenting with acute and chronic pancreatitis.

We are grateful to the referring physicians and surgeons for permission to include some of the cases reported in this study.

\section{References}

Ashton, M. G., Axon, A. T. R., and Lintott, D. J. (1978). Lundh test and ERCP in pancreatic disease. Gut, 19, 910-915.

Baldwin, W. M. (1911). The pancreatic ducts in man, together with a study of the microscopic structure of the minor duodenal papilla. Anatomical Record, 5, 197-228.

Berman, L. G., Prior, J. T., Abramow, S. M., and Ziegler, D. D. (1960). A study of the pancreatic duct system in man by the use of vinyl acetate casts of postmortem preparations. Surgery, Gynecology, and Obstetrics, 110, 391-403.

Cotton, P. B., and Kizu, M. (1977). Malfusion of dorsal and ventral pancreas; a cause of pancreatitis? Gut, 18, A400. (Abstract).

Gregg, J. A. (1977). Pancreas divisum: its association with pancreatitis. American Journal of Surgery, 134, 539-543.

Heiss, F. W., and Shea, J. A. (1978). Association of pancreatitis and variant ductal anatomy. American Journal of Gastroenterology, 70, 158-162.

Howard, J., and Jones, R. (1947). The anatomy of the pancreatic ducts. American Journal of Medical Science, 214, 617-622.

Kleitsch, W. P. (1955). Anatomy of the pancreas: a study with special reference to the duct system. Archives of Surgery, 71, 795-802.

Kruse, A. (1977). Pancreas divisum: a significantly higher incidence in chronic pancreatitis? Scandinavian Journal of Gastroenterology, 12, Suppl. 45, 52. (Abstract).

Mairose, U. B., Wurbs, D., and Classen, M. (1978). Santorini's duct-an insignificant variant from normal or an important overflow valve? Endoscopy, 10, 24-29. 
Meckel, J. F., Jr. (1818). Handbuch der pathologischen Anatomie, Vol. 2, pt. 2. C. H. Reclam: Leipzig.

Mottaleb, A., Kapp, F., Noguera, E. C. A., Kellock, T. D., Wiggins, H. S., and Waller, S. L. (1973). The Lundh test in the diagnosis of pancreatic disease; A review of five years' experience. Gut, 14, 835-841.

Phillip, J., Koch, H., and Classen, M. (1974). Variations and anomalies of the papilla of Vater, the pancreas and the biliary duct system. Endoscopy, 6, 70-77.

Rienhoff, W. F., Jr., and Pickrell, K. L. (1945). Pancreatitis - an anatomic study of the pancreatic and extrahepatic biliary systems. Archives of Surgery, 51, 205-219.

Rösch, W., Koch, H., Schaffner, O., and Demling, L.
(1976). The clinical significance of pancreas divisum. Gastrointestinal Endoscopy, 22, 206-207.

Santorini, G. D. (1775). Anatomici Summi Septemdecim Tabulae. p. 150 and Tabulae XII et XIII. Regia Typographica: Parma.

Thompson, M. H., Williamson, R. C. N., and Salmon, P. R. (1978). Pancreas Divisum: a Cause of Abdominal Pain? Paper presented at XIth Meeting of European Pancreatic Club (abstract).

Wiggins, H. S. (1966). Simple method for estimating trypsin. Gut, 8, 415-416.

Wirsung, J. G. (1642). Figura Ductus Cuiusdam cum Multiplicibus suis Ramulis Noviter in Pancreate in Diversis Corporibus Humanis Observati. Padua. 